\title{
Diagnóstico molecular de candidemia mediante PCR semianidada en pacientes críticos
}

\section{Molecular diagnosis of candidemia by semi- nested PCR in critically ill patients}

\author{
William Pascual Ospina, Jorge Alberto Cortés \\ - Bogotá, D.C. (Colombia)
}

\section{Resumen}

Objetivos: estandarizar una prueba molecular basada en PCR semianidada para el diagnóstico de candidemia

Metodología: se desarrolló un estudio piloto en que se optimizó el protocolo de extracción y utilización de ADN ribosomal, amplificando la región ITS-2 completa de Candida spp. y mediante PCR semianidada, reamplificando segmentos específicos de nueve especies clínicamente relevantes de Candida. Se utilizaron muestras clínicas de plasma de pacientes críticamente enfermos con candidemia documentada microbiológicamente del Hospital de La Samaritana en Bogotá. Se utilizó una muestra de candidemia simulada utilizando una cepa de Candida obtenida de orina.

Resultados: se logró protocolizar dicha técnica y tener a partir de muestras clínicas de plasma una sensibilidad probable de $90 \%$ y especificidad teórica cercana a 100\%. La capacidad de detección de este ensayo es hasta de una célula por $200 \mu 1$ de plasma.

Conclusiones: se estandarizó una prueba molecular para detección de candidemia en pacientes críticamente enfermos (Acta Med Colomb 2011; 36: 135-140).

Palabras clave: (DeCS), Candida, Candida/aislamiento \& purificación, candidemia, candidemia/ diagnóstico, candidemia/microbiology, técnicas de diagnóstico molecular, reacción en cadena de la polimerasa.

\footnotetext{
Abstract

Objectives: to standardize a molecular test based on semi-nested PCR for diagnosis of candidemia.

Methods: we developed a pilot study by means of optimizing the extraction protocol and use of ribosomal DNA, amplifying the complete ITS-2 region of Candida spp. and by semi-nested PCR reamplifying specific segments of nine clinically relevant Candida species. Clinical samples of plasma of critically ill patients with microbiologically proven candidemia from a third level hospital in Bogotá. Another sample of simulated candidemia was used with Candida strain form a urine sample.

Results: we were able to standardize this technique and achieved from clinical samples of plasma a likely sensitivity of $90 \%$ and a theoretical specificity of $100 \%$. The detection capability of this test is up to 1 cell per $200 \mu \mathrm{l}$ of plasma.

Conclusion: a molecular test for detection of candidemia among critically ill patients was standardized (Acta Med Colomb 2011; 36: 135-140).

Key words (MeSH): candida, candida/isolation \& purification, candidemia, candidemialdiagnosis, candidemia/microbiology, molecular diagnostic technology, polymerase chain reaction, nested PCR.
}

Dr. William Pascual Ospina Barragán: Departamento de Medicina, Facultad de Medicina, Universidad Nacional de Colombia; Dr. Jorge Alberto Cortés: Departamento de Medicina, Grupo de Investigación en Enfermedades Infecciosas, Facultad de Medicina, Universidad Nacional de Colombia. Bogotá, D.C. (Colombia).

Correspondencia: Dr. William Pascual Ospina. Bogotá, D.C. (Colombia).

E-mail: wipos@hotmail.com

Recibido: 29/XI/2010 Aceptado: 28/VII/2011

\section{Introducción}

Los adelantos técnicocientíficos en medicina han logrado mejorar la supervivencia de pacientes con enfermedades graves; sin embargo, la exposición prolongada de dichos pacientes al medio hospitalario y una variedad de condi- ciones subyacentes propias del paciente predisponen a la colonización y posterior infección por agentes oportunistas incluyendo diferentes especies de hongos. Las infecciones del torrente sanguíneo por Candida en los pacientes críticamente enfermos determinan un factor de mortalidad que 
se puede elevar por encima de 50\% (1). En Estados Unidos, Candida spp. es la cuarta causa más frecuente de infección del torrente sanguíneo (2) y en Colombia es la quinta entre los pacientes en la unidad de cuidado intensivo (3).

El gold standard de infección por Candida spp. diseminada o invasiva lo constituyen los hemocultivos cuyo rendimiento para mejor los casos es del 50\% (4); esto quiere decir que en más de la mitad de los pacientes infectados por Candida spp. no se logrará documentar la infección micótica o sólo se identificará en forma tardía. La supervivencia de pacientes con candidiasis diseminada depende en gran medida del pronto inicio de antimicóticos y de su actividad frente a la Candida spp, aislada (5). Se ha demostrado que un retardo en el inicio de antimicóticos superior a 12 horas puede relacionarse con un aumento de la mortalidad de forma significativa. Sin embargo, los resultados de los hemocultivos tardan mucho más que aquellos para los agentes bacterianos (6).

Los métodos de detección basados en ADN ofrecen un medio potencialmente más sensible para el diagnóstico de infecciones con baja carga infecciosa. La presencia de ADN de diversos microorganismos patógenos en la sangre de paciente infectados ha sido demostrada. La localización del microorganismo en la sangre es de valor crítico y un paso limitante para definir a partir de qué tipo de muestra se debe trabajar; es decir, para microorganismos de localización intracelular la muestra ideal inicial es sangre total y el material de trabajo será el concentrado de glóbulos rojos y blancos, luego de centrifugación y separación del plasma. En el caso de las infecciones por Candida spp., no existía claridad acerca de la muestra de trabajo inicial para optimizar la localización y posterior purificación de ADN micótico. Hasta el momento diversos protocolos de PCR realizados en líquidos corporales para detectar ADN de Candida han producido resultados algunos satisfactorios y otros no tan alentadores (7). Los trabajos hasta el momento realizados que involucran la amplificación de ADN por PCR se basan en el previo cultivo de sangre y no han probado su eficacia a partir de muestras clínicas directas. Adicionalmente debido al aumento de infecciones por Candida spp. no albicans cuyo perfil de susceptibilidad a los antimicóticos es variable; se quiere disponer de un método diagnóstico que sea completamente sensible y a la vez suficientemente específico y que logre caracterizar a nivel de especie. El objetivo del presente estudio fue el de desarrollar una prueba de PCR para la identificación de Candida en el torrente sanguíneo de pacientes con candidemia.

\section{Material y métodos}

Se trata de un estudio experimental de desarrollo y estandarización de una prueba molecular para aplicar en muestras de plasma.

\section{Muestras}

Se seleccionaron pacientes con hemocultivos positivos para especies de Candida que estaban críticamente enfermos en el Hospital Universitario La Samaritana en Bogotá. Los hemocultivos eran solicitados por los médicos tratantes de acuerdo con con su propia sospecha de infección bacteriana o micótica del torrente sanguíneo. A los pacientes (o sus acudientes) con hemocultivos positivos para una levadura se les informó de la posibilidad de participar en el estudio y se le solicitó consentimiento informado durante el segundo semestre de 2009. Se tomaron dos muestras de sangre total por venopunción simple de pacientes con candidemia diagnosticada, previo al inicio del antimicótico.

También se utilizó una cepa de Candida spp., obtenida a partir de un urocultivo.

Por conveniencia, se seleccionaron los diez primeros pacientes con hemocultivos positivos para realizar la extracción de ADN y la prueba molecular. Los diagnósticos microbiológicos del laboratorio de microbiología se mantuvieron cegados a los investigadores hasta el final.

\section{Aspectos éticos}

Se obtuvo la aprobación por los comités científico y ético del Hospital de La Samaritana E.S.E. de Bogotá D.C.. Cada paciente o su acudiente en casos en los que el paciente no estuviera en capacidad de dar su consentimiento fue informado del estudio y nuevas muestras de sangre fueron tomadas previo al inicio del antimicótico.

\section{Procedimientos de laboratorio}

Se tomaron $10 \mathrm{~mL}$ de sangre en tubos de vacío con EDTA. Estas muestras se llevan a centrifugación a 1000 g/ min para separar la fase celular y de plasma. Se toma el sobrenadante con micropipeta evitando la fase celular y obteniendo muestras de plasma de estos pacientes de 4-6 mL de cada uno, dado que es la muestra que teóricamente tiene mejor rendimiento de detección de candidemia por PCR (8). Estas muestras se transportaron de forma independiente, debidamente rotuladas y refrigeradas al laboratorio de biología molecular de la Fundación Instituto de Inmunología de Colombia, FIDIC, para su almacenamiento en frascos cerrados con rosca a $-20^{\circ} \mathrm{C}$

El almacenaje en promedio duro seis meses hasta el momento del análisis molecular de las 10 muestras recolectadas.

Los primers a utilizar son: dos primers universales CTSF (5'-TCGCATCGATGAAGAACGCAGC-3') y CTSR (5'-TCTTTTCCTCCGCTTATTGATATGC-3') para amplificar la región ITS 2 entera. Luego se utilizarán primers directos, específicos para nueve especies de Candida anidados en el extremo $3^{\prime}$ suministrados por la FIDIC, no mostrados.

Los resultados se consideran positivos con la aparición de una o varias bandas de ADN marcado, del tamaño esperado y visualizado con luz ultravioleta en comparación con marcadores de tamaño molecular de 100 pares de bases de ADN.

La sensibilidad esperada según los conceptos teóricos debe superar $80 \%$ y la especificidad será cercana a $100 \%$ con la capacidad de identificar nueve especies de Candida spp., clínicamente relevantes. 
Simultáneamente se decide para efecto de control de calidad y estandarización, fabricar muestras de candidemia simulada (9), a partir de muestras de plasma (una) de los investigadores y cultivo de Candida spp., de aislamiento de orina suministrado también por el laboratorio de microbiología del Hospital Universitario de La Samaritana. Este cultivo se transportó en fase sólida al laboratorio de biología molecular del Instituto, en donde se replica para cultivo líquido el cual fue cuantificado por método de estándares de turbidez de McFarland, para luego hacer diluciones seriadas y mezcla con el plasma de los investigadores hasta obtener candidemias simuladas del orden de $5 \times 10^{5}, 5 \times 10^{3}, 5 \times 10^{1}$ y 5 células por $\mu 1$ de plasma. Estas candidemias simuladas sirvieron para estandarizar la técnica de extracción de ADN.

El protocolo de extracción final incluye:

1. Tomar $1.8 \mathrm{~mL}$ de plasma infectado en tubos de $2 \mathrm{~mL} \mathrm{y}$ centrifugar a $10000 \mathrm{~g} / \mathrm{min}$ a temperatura ambiente.

2. Descartar el sobrenadante y resuspender el pellet en 300 $\mu 1$ de la solución Microbead UltraClean ${ }^{\circledR}$ Microbial ADN Isolation Kit de (MO BIO laboratories Inc.). Este kit se escogió por incluir un paso de lisis celular químico optimizado por el efecto mecánico de microcápsulas. Continuar el protocolo según el fabricante.

Es importante en el momento de utilizar SDS incubar la muestra a $65^{\circ} \mathrm{C}$ durante 10 minutos antes de continuar. $\mathrm{Al}$ final del protocolo el filtro se desecha y quedamos con una muestra de aproximadamente 40-50 $\mu 1$ de ADN en Tris 10 $\mathrm{mM}$ pH8 que no contiene EDTA el cual es guardado $-20^{\circ} \mathrm{C}$.

Las condiciones de PCR inicial son:

Se utilizan los primers descritos previamente (10) CTSF y CTSR, directo y reverso, respectivamente a concentraciones finales de 10 pmol buffer $1 \mathrm{X}, \mathrm{MgCl} 21.5 \mathrm{mM}$, dNTPs 0.1 $\mathrm{mM}$, Taq ADN polimerasa a razón de 0.5 unidades por 10 $\mu 1,2 \mu 1$ de ADN extraído en una reacción total de $10 \mu 1$ con los siguientes ciclos: $94^{\circ} \mathrm{C} 3$ minutos por un ciclo; 35 ciclos de tres temperaturas así: $94^{\circ} \mathrm{C}$ un minuto, $60^{\circ} \mathrm{C} 30$ segundos y $72^{\circ} \mathrm{C}$ un minuto y un ciclo final de $72^{\circ} \mathrm{C}$ por 10 minutos. Ciclo final de seguridad a $4^{\circ} \mathrm{C}$.

La PCR semianidada se realiza con CTSR descrito y 9 primers directos de nueve especies clínicamente relevantes de Candida spp. a saber: C. albicans, C.tropicalis, C.glabrata, C. parapsilosis, C. famata, C. krusei, C. guilliermondii, $C$. dubliniensis and C. lusitaniae (10)( FIDIC). Las condiciones fueron las siguientes: primers $5 \mathrm{pM}$, buffer $1 \mathrm{X}, \mathrm{MgCl} 2$ 1,5 $\mathrm{mM}$, dNTPs $0.1 \mathrm{mM}$, Taq DNA polimerasa 0.5 unidades, 1 $\mu 1$ del amplificado de DNA inicial diluido 1:10, en un volumen total de $10 \mu 1$, realizando el siguiente ciclado: Un ciclo $94^{\circ} \mathrm{C}$ por $3 \mathrm{~min}$; 20 ciclos de tres temperaturas asi: $94^{\circ} \mathrm{C}$ un minuto, $60^{\circ} \mathrm{C} 30$ segundos y $72^{\circ} \mathrm{C}$ un minuto; y un ciclo de final de extensión de $72^{\circ} \mathrm{C} 10$ minutos.

\section{Resultados}

$\mathrm{Al}$ momento del primer gel de electroforesis en agarosa al 1\% para ADN genómico, no logramos visualizar ADN de Candida spp. en las 10 muestras clínicas, pero se logra visualizar DNA genómico en las muestras de candidemia simulada (concentradas mediante Speedvac) del orden de $5 \times 10^{5}$ y 5 células $/ \mathrm{mL}$; sin lograr visualizar el mismo en las muestras de $5 \times 10^{3}$ y $5 \times 10^{1}$ suponiendo degradación o pérdida de DNA en estas últimas muestras.

A pesar de este resultado negativo en el gel de electroforesis de ADN genómico de Candida spp., en muestra clínicas, se decide continuar con el protocolo para amplificación del producto de ITS2 completo por PCR en las muestras clínicas dado que la concentración probable esperada de ADN es mínima tal como ocurre con las bajas candidemias habituales las cuales son positivas para hemocultivos sólo en la mitad de los casos.

El resultado de este producto inicial de PCR amplificando la región completa de ITS2 se logra visualizar en siete de las 10 muestras; sin embargo, la muestra identificada como \#2 amplifica claramente dos bandas, lo cual se puede interpretar como inespecificidad de la prueba o la presencia de dos amplicones positivos de diferente tamaño y provenientes de dos especies diferentes de Candida spp. Amplifican también con la primera PCR las muestras de candidemia simulada identificadas como \#11 y \#14 del orden de $5 \times 10^{5}$ y 5 células $/ \mathrm{mL}$ respectivamente. No amplifica la PCR de la muestra identificada como \#13 de candidemia simulada por lo cual se decide descartar las muestras \#12 y \#13 del orden $5 \times 10^{3}$ y $5 \times 10^{1}$ respectivamente, confirmando la ausencia de ADN íntegro en estas mismas. Los respectivos controles negativos no amplificaron (fotografía omitida por mala calidad, pero registrada).

Con este resultado tenemos una sensibilidad de la PCR a partir de sangre de pacientes con candidemia de $70 \%$ y para descartar la inespecificidad de la prueba se hace PCR semianidada de la muestra clínica \#5, que fue el amplicon mejor visualizado y de la muestra clínica \#2, que es la que presenta dos ampliaciones se realiza inicialmente la PCR seminidada para estas dos muestras.

El resultado de este ensayo con las muestras clínicas \#2 y \#5 inicial amplifica con los primers para C. guilliermondii (primer directo específico) y C.famata /guilliermondii (este primer ampifica para ambas especies) en ambas muestras, lo cual corresponde a la presencia de $C$. guilliermondii en las dos muestras (Figuras 1 y 2). Sin embargo, al tener la amplificación inicial de la PCR con dos bandas en la segunda muestra y amplificar con los dos sets de primers se asume que se encuentran presentes las dos especies de Candida en la muestra dos.

$\mathrm{Al}$ descartar la posibilidad de inespecificidad de la prueba se procede entonces a realizar PCR semianidada de las ocho muestras clínicas restantes $(1,3,4,6,7,8,9$ y \#10) y de las dos muestras de candidemia simulada óptimas descritas (\#11, \#14), bajo las mismas condiciones anteriormente descritas y con sus respectivos controles negativos (Figuras $3,4$ y 5$)$.

El resultado de esta PCR es analizado de la siguiente manera: para C. lusitaniae amplifica la muestra clínica \#8; 


\section{E8 E10 E6 D10 D12 E12 D8 D6 D4 CN M}

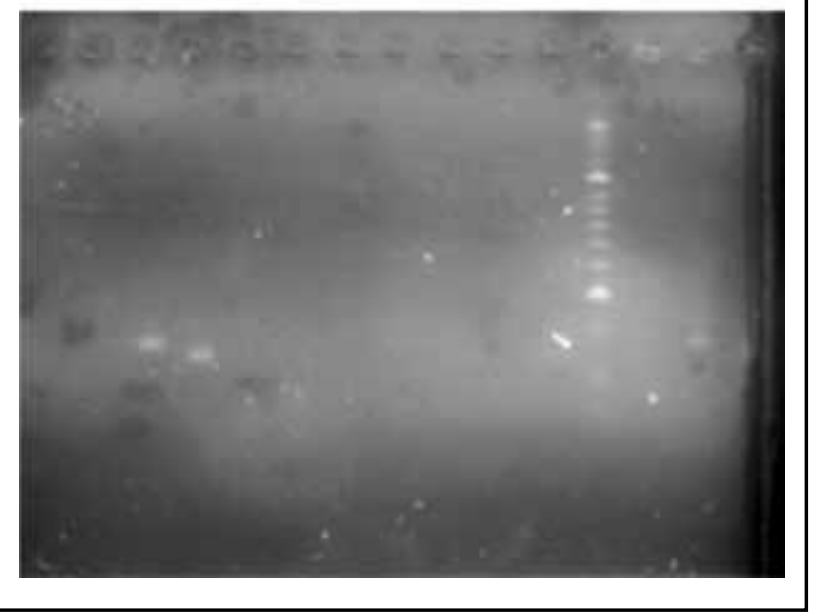

Figura 1. PCR semianidada de la muestra 2 amplificando con primers de C. guilliermondii (E10) y primers de C. famata/guilliermondii (E6). No amplifican E8 C. lusitaniae; D10 C. krusei; D12 C. glabrata; E12 C dubliniensis; D8 C. parapsilosis; D6 C. tropicalis; D4 C. albicans. $M$ son los marcadores de peso molecular y CN control negativo. Los códigos de letras y números así como su respectivo color asignado a los primers se especifican en la Figura 5.

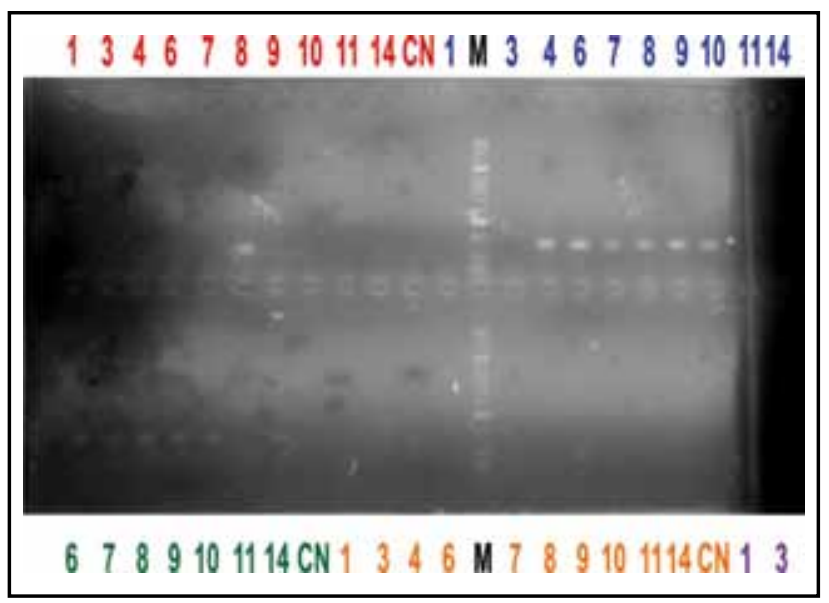

Figura 3. PCR semianidada en las muestras clínicas del 1 al 10 y controles negativos. Se omiten muestras 2 y 5 antes analizadas; se adicionan las muestras 11 y 14 de candidemia simulada y control negativo para cada set de primers. C. lusitaniae amplifica la muestra clínica \#8; para C. guilliermondii amplifican las muestras clínicas \#4, \#6, 7, 8, 9 y \#10 y la candidemia simulada \# 14; para C. famata/guilliermondii amplifican las muestra clínicas \#6, \#7,8,9 y 10 y la candidemia simulada \#14 nuevamente; no hay resultado de amplificación para C. krusei ni para C. glabrata. Los códigos de letras y números así como su respectivo color asignado a los primers se especifican en la Figura 5.

para $C$. guilliermondii amplifican las muestras clínicas \#4, \#5, 7, 8, 9 y \#10 y la candidemia simulada \# 14; para $C$. famata/guilliermondii amplifican las muestra clínicas \#6, \#7, 8,9 y \#10 y la candidemia simulada \#14 nuevamente; no hay resultado de amplificación para $C$. krusei ni para C. glabrata.

C. dubliniensis amplifica en las muestras \#1 y \#7.

C. parapsilosis y $C$. tropicalis no tiene amplificados y curiosamente al contrario de lo ampliamente esperado no hay amplificación para $C$. albicans.

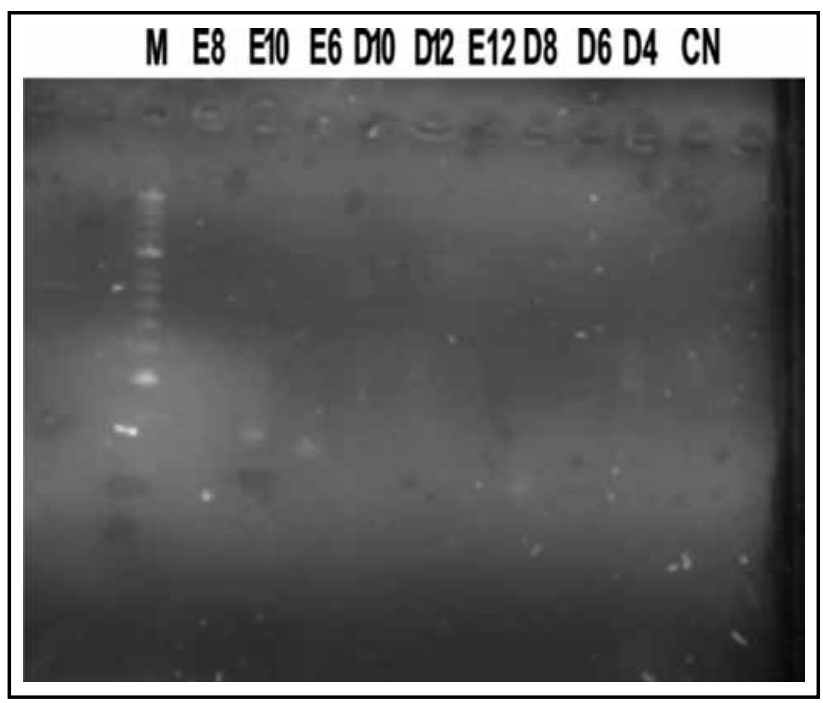

Figura 2. PCR semianidada de la muestra 5 amplificando con primers de C. guilliermondi (E10) y primers de C. famata/guilliermondii (E6). No amplifican E8 C. lusitaniae; D10 C. krusei; D12 C. glabrata; E12 C dubliniensis; D8 C. parapsilosis; D6 C. tropicalis; D4 C. albicans. $M$ son los marcadores de peso molecular y CN control negativo. Los códigos de letras y números así como su respectivo color asignado a los primers se especifican en la Figura 5.

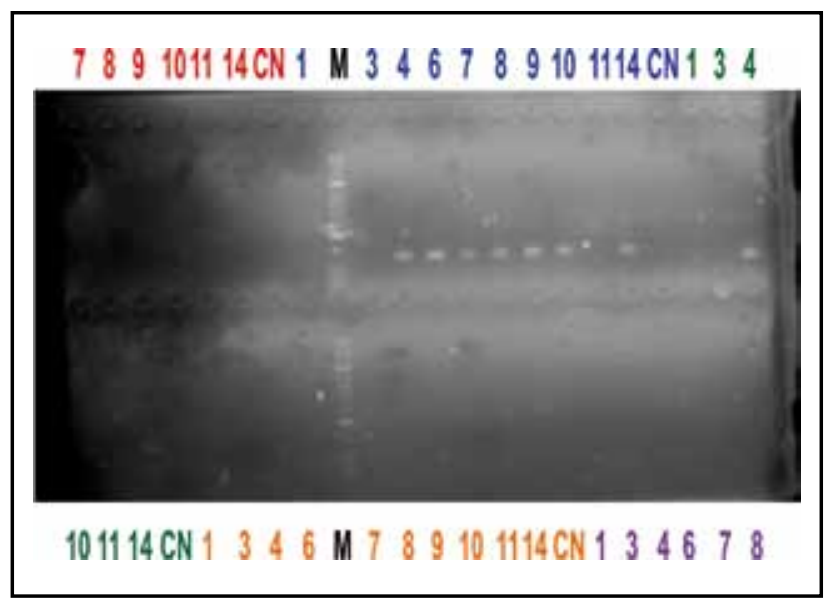

Figura 4. C. lusitaniae amplifica la muestra clínica \#8; para C. guilliermondii amplifican las muestras clínicas \#4, \#6, 7, 8, 9 y \#10 y la candidemia simulada \# 14; para C. famatal guilliermondii amplifican las muestra clínicas \#6, \#7, 8, 9 y \#10 y la candidemia simulada \#14 nuevamente; no hay resultado de amplificación para C. krusei ni para C. glabrata. Se trata de la continuación de la foto anterior. Los códigos de letras y números así como su respectivo color asignado a los primers se especifican en la Figura 5.

La Tabla 1 muestra los resultados obtenidos por el laboratorio de microbiología y por la técnica de PCR semianidada.

\section{Discusión}

Las limitaciones de los sistemas de hemocultivo para la detección de fungemia han llamado la necesidad de desarrollar métodos alternativos de diagnóstico que sean rápidos, sencillos y precisos. La PCR semianidada a partir de ADN extraído por métodos de lisis y purificado mediante 


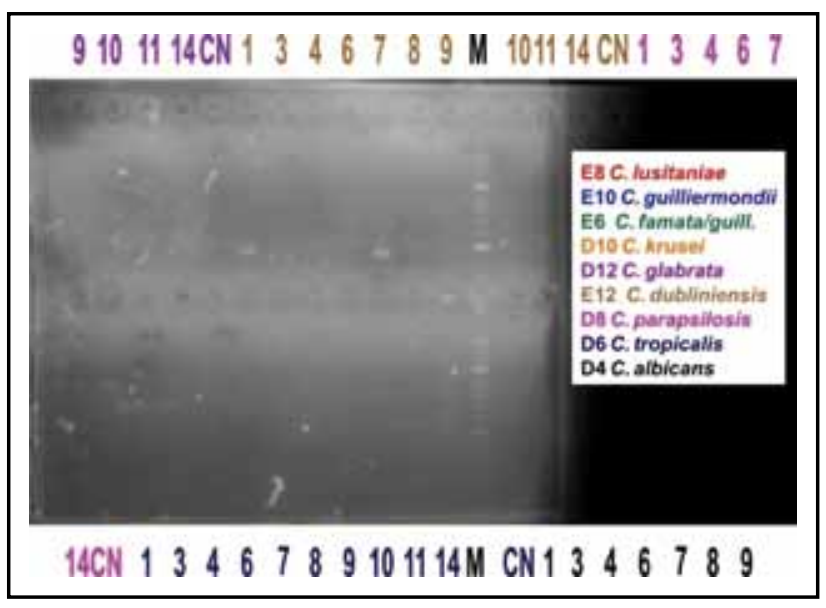

Figura 5. C. dubliniensis amplifica en la muestra \#1 y \#7. C. parapsilosis, C. tropicalis y C. albicans no tiene amplificados. Los códigos de letras y números así como el respectivo color asignado a los primers se especifican en esta figura.

precipitación tiene la posibilidad de un mejor desempeño que los hemocultivos para candidemia y más aún si se realiza a partir de plasma o suero, de esta manera logrando un diagnóstico acelerado a nivel de especie. Un estudio utilizando el sistema de hemocultivos BACT/Alert mostró que el tiempo promedio de crecimiento de Candida varió dependiendo de la especie entre 26.2 y 80 horas, con un tiempo promedio de reporte sin identificación de especie entre 28.7 y 98 horas (6). Esto significaría que una prueba molecular podría abreviar el tiempo de reporte a cerca de 24 horas (o menos) con un ahorro de hasta tres días.

Para nuestra prueba piloto podemos concluir que el diagnóstico molecular de candidemia lo podemos tener para la primera PCR alrededor de ocho horas posterior a la toma de la muestra, si se tiene implementado un laboratorio de biología molecular y de procesamiento inmediato de la misma, con una sensibilidad inicial de al menos $70 \%$ sin dar resultados a nivel de especie. Esta sensibilidad es relativamente baja, si es comparada frente a $100 \%$, que para este estudio corresponde al resultado de los hemocultivos, y debe ser evaluada en pacientes con factores de riesgo para candidemia y que tienen hemocultivos negativos en los cuales se espera mayor rendimiento de la prueba molecular y para quienes sería de la mayor utilidad clínica. Resultados similares con respecto a una menor sensibilidad cuando se trabaja a partir de resultados positivos de microbiología se han obtenido en otros modelos experimentales como es el ejemplo de tuberculosis meníngea (12). Una vez utilizada la metodología semianidada se obtuvo una sensibilidad probable de $90 \%$ con la ventaja de la especificidad a nivel de especie y con una sensibilidad de detección de hasta una célula /200 $\mu 1$ de plasma.

La sensibilidad global de la PCR semianidada a partir de muestras de hemocultivo debe estar por encima de $90 \%$. Se deben adelantar pruebas similares en pacientes con factores de riesgo para candidemia y determinar así la sensibilidad
Tabla 1. Resultados del laboratorio de microbiología de la PCR.

\begin{tabular}{|c|c|c|}
\hline Muestra & $\begin{array}{c}\text { Identificación } \\
\text { por microbiología }\end{array}$ & $\begin{array}{c}\text { Identificación } \\
\text { por PCR }\end{array}$ \\
\hline 1 & C.famata & C.dubliniensis \\
2 & C.famata & C.famata y C.guilliermondii \\
3 & C.albicans & No amplificada \\
4 & C.parapsilosis & C.guilliermondii \\
5 & C.parapsilosis & C.guilliermondii \\
6 & C.albicans & C.guilliermondii \\
7 & C.parapsilosis & C.guilliermondii y C.dubliniensis \\
8 & Candida spp. & C.lusitaniae y C.guilliermondii \\
9 & C.tropicalis & C.guilliermondii \\
10 & C.albicans & C.guilliermondii \\
Muestra orina & C.guilliermondii & C.guilliermondii \\
\hline
\end{tabular}

real para el diagnóstico y compararla de manera independiente al resultado de los hemocultivos. La ventaja primordial de realizar el diagnóstico molecular de candidemia por PCR semianidada, radica en un diagnóstico temprano (aproximadamente 14 horas luego de tomada la muestra) y la determinación temprana de la especie, lo cual brindará una herramienta para guiar el tratamiento antimicótico de la especie en particular de acuerdo con los lineamientos terapéuticos ya establecidos (13).

Se encontró discordancia entre los resultados del laboratorio de microbiología y de la técnica molecular. Esto se puede explicar por varias razones: una sería la falta de especificidad de la prueba para la identificación de las diversas especies por errores de la técnica microbiológica o de la técnica molecular (14); otra debido a errores en la interpretación de los resultados obtenidos y de los patrones de amplificación. También existe la posibilidad de la presencia de un brote de C. guilliermondii en el interior de las unidades de cuidado intensivo estudiadas. Finalmente, estos resultados podrían corresponder a contaminación de la prueba con ADN de la cepa utilizada para la candidemia simulada, aunque esta última es menos probable ya que los controles negativos se mantuvieron durante todos los ensayos.

Otra ventaja que claramente es evidente del análisis molecular, es el hecho de facilitar el diagnóstico de coinfección por dos o más especies de Candida, lo cual se puede ver en $30 \%$ de los casos y ha sido reportado previamente (15), lo cual predice un probable foco abdominal, la necesidad de ampliar cubrimiento antimicótico y de igual forma puede explicar el fallo terapéutico en algunas ocasiones.

La cantidad de muestras para este estudio nos permitió estandarizar las condiciones para el estudio de biología molecular a partir de muestras clínicas; sin embargo, es insuficiente para el cálculo de medidas de precisión diagnóstica como sensibilidad, especificidad, valores predictivos, razón de probabilidad y medidas de correlación con otras pruebas y 
de validez interna y externa. Para esto se requiere un estudio clínico de mayor magnitud cuya dificultad principal estriba en la construcción de estándar de oro apropiado.

Las unidades de alta tecnología y de elevados costos como las unidades de cuidado intensivo deben considerar la utilización de pruebas de diagnóstico rápido y certero como aquella potencialmente brindada por métodos basados en la biología molecular, especialmente en escenarios como el de candidemia con una alta morbilidad y mortalidad (16). Se plantea dada la utilidad de la PCR a partir de muestras clínicas de esta técnica; hacer un esfuerzo por los grupos del país con investigación en Candidemia para hacer un estudio de diagnóstico en pacientes con factores de riesgo alto de candidemia en UCI y determinar la sensibilidad y especificidad de la prueba en este contexto.

\section{Agradecimientos}

Queremos agradecer el apoyo técnico y científico prestado por el doctor Manuel Alfonso Patarroyo y otros colaboradores de la Fundación Instituto de Inmunología de Colombia, FIDIC. También el apoyo de la doctora Anita Montañez, coordinadora del Grupo de Investigación de la Facultad de Medicina.

Este estudio fue realizado en el Hospital Universitario de La Samaritana, gracias al apoyo y la labor técnica de la doctora Lucy Guzmán.

Este proyecto se realizó con apoyo financiero de la Universidad Nacional de Colombia a través de la modadlidad de apoyo a tesis de posgrado de la Convocatoria Nacional de Investigación de 2009 (código 9974).

No hay conflictos de interés que declarar por parte de los investigadores.

\section{Referencias}

1. Prowle JR, Echeverri JE, Ligabo EV, Sherry N, Taori GC, Crozier TM, et al. Acquired bloodstream infection in the intensive care unit: incidence and attributable mortality. Crit Care 2011; 15: R100.

2. Edmond MB, Wallace SE, McClish DK, Pfaller MA, Jones RN, Wenzel RP. Nosocomial bloodstream infections in United States hospitals: a three-year analysis. Clin Infect Dis 1999; 29: 239-44.

3. Cortes JA, Reyes P, Gomez C, Buitrago G, Leal AL. Fungal bloodstream infections in tertiary care hospitals in Colombia. Rev Iberoam Micol 2011; 28: 74-8.

4. Kami M, Machida U, Okuzumi K, Matsumura T, Mori Si S, Hori A, et al.
Effect of fluconazole prophylaxis on fungal blood cultures: an autopsy-based study involving 720 patients with haematological malignancy. Br J Haematol 2002; 117: 40-6

5. Patel GP, Simon D, Scheetz M, Crank CW, Lodise T, Patel N. The effect of time to antifungal therapy on mortality in Candidemia associated septic shock. Am J Ther 2009; 16: 508-11.

6. Fernandez J, Erstad BL, Petty W, Nix DE. Time to positive culture and identification for Candida blood stream infections. Diagn Microbiol Infect Dis 2009; 64: $402-7$.

7. White PL, Barton R, Guiver M, Linton CJ, Wilson S, Smith M, et al. A consensus on fungal polymerase chain reaction diagnosis?: a United Kingdom-Ireland evaluation of polymerase chain reaction methods for detection of systemic fungal infections. J Mol Diagn 2006; 8: 376-84.

8. Bougnoux M, Dupont C, Mateo J, Saulnier P, Faivre V, Payen D, et al. Serum is more suitable than whole blood for diagnosis of systemic candidiasis by nested PCR. J Clin Microbiol 1999; 37: 925-30.

9. Maaroufi Y, De Bruyne JM, Duchateau V, Georgala A, Crokaert F. Early detection and identification of commonly encountered Candida species from simulated blood cultures by using a real-time PCR-based assay. J Mol Diagn 2004; 6: 108-14

10. Ahmad S, Khan Z, Mustafa AS, Khan ZU. Seminested PCR for diagnosis of candidemia: comparison with culture, antigen detection, and biochemical methods for species identification. J Clin Microbiol 2002; 40: 2483-9.

11. Bougnoux ME, Kac G, Aegerter P, d'Enfert C, Fagon JY. Candidemia and candiduria in critically ill patients admitted to intensive care units in France: incidence, molecular diversity, management and outcome. Intensive Care Med 2008; 34: 292-9.

12. Dora JM, Geib G, Chakr R, Paris F, Mombach AB, Lutz L, et al. Polymerase chain reaction as a useful and simple tool for rapid diagnosis of tuberculous meningitis in a Brazilian tertiary care hospital. Braz J Infect Dis 2008; 12: 245-7.

13. Pappas PG, Kauffman CA, Andes D, Benjamin DK, Jr., Calandra TF, Edwards JE, Jr, et al. Clinical practice guidelines for the management of candidiasis: 2009 update by the Infectious Diseases Society of America. Clin Infect Dis 2009; 48: 503-35.

14. Desnos-Ollivier M, Ragon M, Robert V, Raoux D, Gantier JC, Dromer F. Debaryomyces hansenii (Candida famata), a rare human fungal pathogen often misidentified as Pichia guilliermondii (Candida guilliermondii). J Clin Microbiol 2008; 46: 3237-42.

15. Ortega M, Marco F, Soriano A, Almela M, Martinez JA, Lopez J, et al. Candida species bloodstream infection: epidemiology and outcome in a single institution from 1991 to 2008. J Hosp Infect 2011; 77: 157-61.

16. Morgan J, Meltzer MI, Plikaytis BD, Sofair AN, Huie-White S, Wilcox S, et al. Excess mortality, hospital stay, and cost due to candidemia: a case-control study using data from population-based candidemia surveillance. Infect Control Hosp Epidemiol 2005; 26: 540-7. 